\title{
Infinitely many solutions to superlinear second order $m$-point boundary value problems
}

Ruyun $\mathrm{Ma}^{1 *}$, Chenghua Gao ${ }^{1}$ and Xiaoqiang Chen ${ }^{2}$

* Correspondence: mary@nwnu. edu.cn

${ }^{1}$ Department of Mathematics, Northwest Normal University, Lanzhou, 730070, PR China

Full list of author information is available at the end of the article

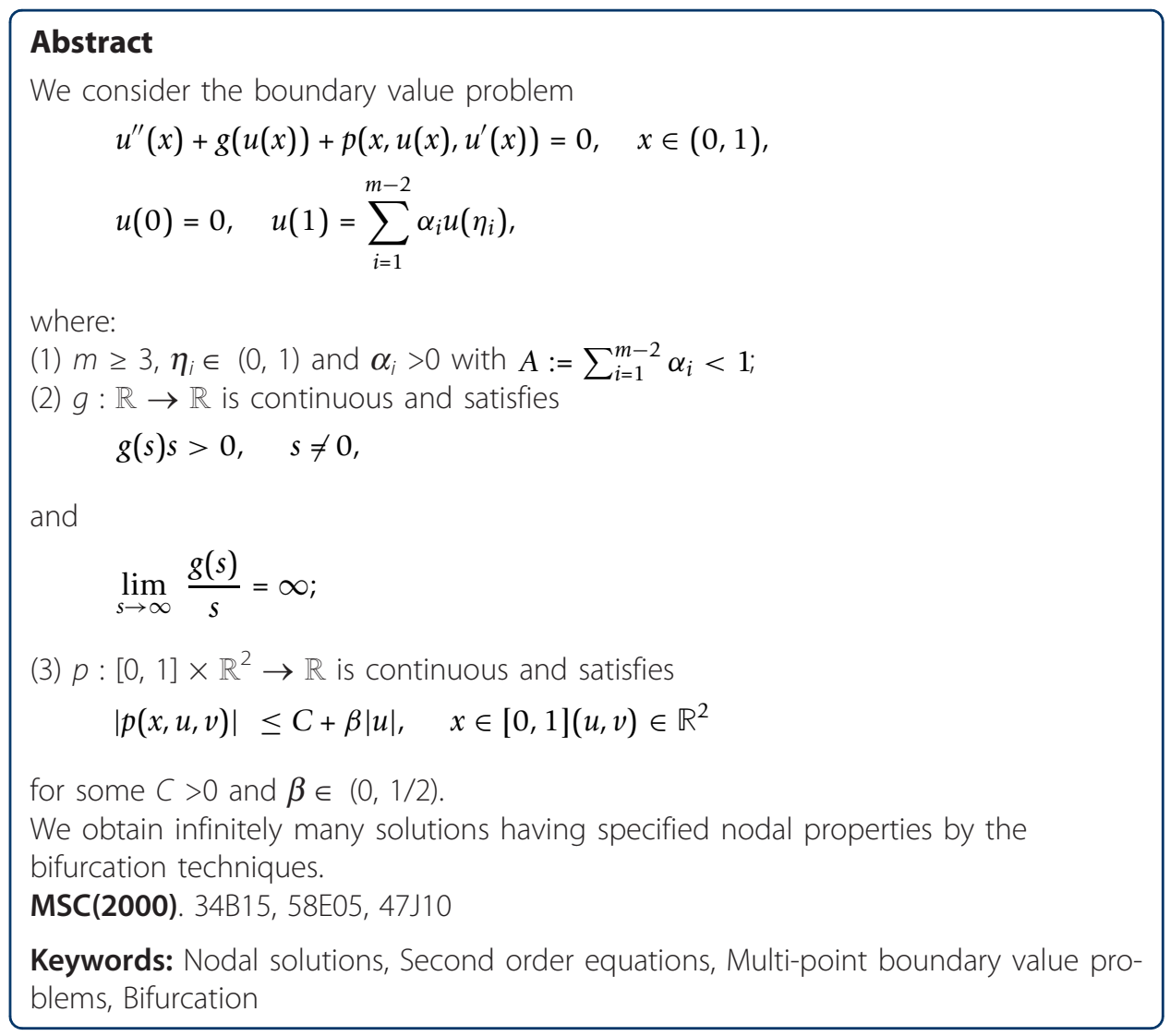

\section{Introduction}

We consider the nonlinear boundary value problem

$$
\begin{aligned}
& u^{\prime \prime}(x)+g(u(x))+p\left(x, u(x), u^{\prime}(x)\right)=0, \quad x \in(0,1), \\
& u(0)=0, \quad u(1)=\sum_{i=1}^{m-2} \alpha_{i} u\left(\eta_{i}\right),
\end{aligned}
$$

where 
(H1) $m \geq 3, \eta_{i} \in(0,1)$ and $\alpha_{i}>0$ with

$$
A:=\sum_{i=1}^{m-2} \alpha_{i}<1 ;
$$

(H2) $g: \mathbb{R} \rightarrow \mathbb{R}$ is continuous and satisfies

$$
g(s) s>0, \quad s \neq 0,
$$

and

$$
\lim _{s \rightarrow \infty} \frac{g(s)}{s}=\infty
$$

(H3) $p:[0,1] \times \mathbb{R}^{2} \rightarrow \mathbb{R}$ is continuous and satisfies

$$
|p(x, u, v)| \leq C+\beta|u|, \quad x \in[0,1],(u, v) \in \mathbb{R}^{2}
$$

for some $C>0$ and $\beta \in(0,1 / 2)$.

In order to state our results, we first recall some standard notations to describe the nodal properties of solutions. For any integer, $n \geq 0, C^{n}[0,1]$ will denote the usual Banach space of $n$-times continuously differentiable functions on $[0,1]$, with the usual sup-type norm, denoted by $\|\cdot\| n$. Let $X:=\left\{u \in C^{2}[0,1]: u\right.$ satisfies $\left.(1.2)\right\}, Y:=C^{0}[0$, $1]$, with the norms $|\cdot|_{2}$ and $|\cdot|_{0}$, respectively. Let

$$
E:=\left\{u \in C^{1}[0,1]: u \text { satisfies }(1.2)\right\},
$$

with the norms $|\cdot|_{E}$.

We define a linear operator $L: X \rightarrow Y$ by

$$
L u:=-u^{\prime \prime}, \quad u \in X .
$$

In addition, for any continuous function $g: \mathbb{R} \rightarrow \mathbb{R}$ and any $u \in Y$, let $g(u) \in Y$ denote the function $g(u(x)), x \in[0,1]$.

Next, we state some notations to describe the nodal properties of solutions of (1.1), see [1] for the details. For any $C^{1}$ function $u$, if $u\left(x_{0}\right)=0$, then $x_{0}$ is a simple zero of $u$, if $u^{\prime}\left(x_{0}\right) \neq 0$. Now, for any integer $k \geq 1$ and any $v \in\{+,-\}$, we define sets $S_{k}^{v}, \Gamma_{k}^{v} \subset C^{2}[0,1]$ consisting of the set of functions $u \in C^{2}[0,1]$ satisfying the following conditions:

$$
\underline{S_{k}^{v}}
$$

(i) $u(0)=0, v u^{\prime}(0)>0$; (ii) $u$ has only simple zeros in $[0,1]$ and has exactly $k$ - 1 zeros in $(0,1)$.

\section{$\underline{\Gamma_{k}^{v}}$}

(i) $u(0)=0, v u^{\prime}(0)>0$; (ii) $u^{\prime}$ has only simple zeros in $(0,1)$ and has exactly $k$ such zeros; (iii) $u$ has a zero strictly between each two consecutive zeros of $u$ '.

Remark 1.1 If we add the restriction $u^{\prime}(1) \neq 0$ on the functions in $\Gamma_{k}^{v}$ then $\Gamma_{k}^{v}$ becomes the set $T_{k}^{v}$, which used in [1]. The reason we use $\Gamma_{k}^{v}$ rather than $T_{k}^{v}$ is that the Equation (1.1) is not autonomous anymore.

In [1, Remarks 2.1 and 2.2], Rynne pointed out that 
a. If $u \in T_{k}^{v}$, then $u$ has exactly one zero between each two consecutive zeros of $u$, and all zeros of $u$ are simple. Thus, $u$ has at least $k-1$ zeros in $(0,1)$, and at most $k$ zeros in $(0,1]$;

b. The sets $T_{k}^{v}$ are open in $X$ and disjoint;

c. When considering the multi-point boundary condition (1.2), the sets $T_{k}^{v}$ are in fact more appropriate than the sets $S_{k}^{v}$.

The main result of this paper is the following

Theorem 1.1 Let (H1)-(H3) hold. Then there exists an integer $k_{0} \geq 1$ such that for all integers $k \geq k_{0}$ and each $v \in\{+,-\}$ the problem (1.1), (1.2) has at least one solution $u_{k}^{v} \in \Gamma_{k}^{v}$

Superlinear problems with classical boundary value conditions have been considered in many papers, particularly in the second and fourth order cases, with either periodic or separated boundary conditions, see for example [2-11] and the references therein. Specifically, the second order periodic problem is considered in [2,3], while [4-7] consider problems with separated boundary conditions, and results similar to Theorem 1.1 were obtained in each of these papers. The fourth order periodic problem is considered in [8-10]. Rynne [11] and De Coster [12] consider some general higher order problems with separated boundary conditions also.

Calvert and Gupta [13] studied the superlinear three-point boundary value problem

$$
\begin{aligned}
& u^{\prime \prime}(x)+g(u(x))+p\left(x, u(x), u^{\prime}(x)\right)=0, \quad x \in(0,1), \\
& u(0)=0, \quad u(1)=\beta u(\eta),
\end{aligned}
$$

(which is a nonlocal boundary value problem), under the assumptions:

(A0) $\beta \in(0,1) \cup(1, \infty)$;

(A1) $g: \mathbb{R} \rightarrow \mathbb{R}$ is continuous and satisfies $g(s) s>0, s \neq 0, \frac{g(s)}{s}$ is increasing and

$$
\lim _{|s| \rightarrow \infty} \frac{g(s)}{s}=\infty ;
$$

(A2) $p:[0,1] \times \mathbb{R}^{2} \rightarrow \mathbb{R}$ is a function satisfying the Carathéodory conditions and satisfies

$$
|p(x, u, v)| \leq M_{1}(t, \max (|u|,|v|)), \quad x \in[0,1],(u, v) \in \mathbb{R}^{2},
$$

where $M_{1}:[0,1] \times[0, \infty) \rightarrow[0, \infty)$ satisfies the condition: for each $s \in[0, \infty), M_{1}($, $s)$ is integrable on $[0,1]$ and for each $t \in[0,1], M_{1}(t, \cdot)$ is increasing on $[0, \infty)$ with $s^{-1} \int_{0}^{1} M_{1}(t, s) \mathrm{d} s \rightarrow 0$ as $s \rightarrow \infty$.

Calvert and Gupta used Leray-Schauder degree and some ideas from Henrard [14] and Cappieto et al. [5] to prove the existence of infinity many solutions for (1.7), (1.8). Their results extend the main results in [14].

It is the purpose of this paper to use the global bifurcation theorem, see [15] and [1], to obtain infinity many nodal solutions to $m$-point boundary value problems (1.1), (1.2) under the assumptions (H1)-(H3). Obviously, our conditions (H2) and (H3) are much weaker than the corresponding restrictions imposed in [13]. Our paper uses some of ideas of Rynne [10], which deals with fourth order two-point boundary value problems. By the way, the proof [10, Lemma 2.8] contains a small error (since $\left.|| u^{\prime \prime}\right|_{0} \geq \zeta_{4}(0) \otimes \mid u$ $\left."\right|_{0} \geq \zeta_{4}(R)$ there). So, we introduce a new function $\chi$ (see (3.7)) with 


$$
\chi(0) \geq \zeta_{2}(R)
$$

which are required in applying Lemma 3.4.

\section{Eigenvalues of the linear problem}

First, we state some preliminary results related to the linear eigenvalue problem

$$
L u=\lambda u, \quad u \in X .
$$

Denote the spectrum of $L$ by $\sigma(L)$. The following spectrum results on (2.1) were established by Rynne [1], which extend the main result of Ma and O'Regan [16].

Lemma 2.1. [1, Theorem 3.1] The spectrum $\sigma(L)$ consists of a strictly increasing sequence of eigenvalues $\lambda_{k}>0, k=1,2, \ldots$, with corresponding eigenfunctions $\phi_{k}(x)=\sin \left(\lambda_{k}^{1 / 2} x\right)$. In addition,

(i) $\lim _{k \rightarrow \infty} \lambda_{k}=\infty$;

(ii) $\phi \in T_{k}^{v}$, for each $k \geq 1$, and $\varphi_{1}$ is strictly positive on $(0,1)$.

Lemma 2.2 [1, Theorem 3.8] For each $k \geq 1$, the algebraic multiplicity of the characteristic value $\lambda_{k}$ of $L^{-1}: Y \rightarrow Y$ is equal to 1 .

\section{Proof of the main results}

For any $u \in X$, we define $e(u)(\cdot):[0,1] \rightarrow \mathbb{R}$ by

$$
e(u)(x)=p\left(x, u(x), u^{\prime}(x)\right), \quad x \in[0,1] .
$$

It follows from (1.5) that

$$
|e(u)(x)| \leq C+\beta|u(x)|, \quad x \in[0,1] .
$$

For any $s \in \mathbb{R}$, let

$$
G(s)=\int_{0}^{s} g(\tau) \mathrm{d} \tau \geq 0,
$$

and for any $s \geq 0$, let

$$
\gamma(s)=\max \{|g(r)|:|r| \leq s\}, \quad \Gamma(s)=\max \{G(r):|r| \leq s\} .
$$

We now consider the boundary value problem

$$
u^{\prime \prime}+\lambda u+\alpha(g(u)+e(u))=0, \quad u \in X,
$$

where $\alpha \in[0,1]$ is an arbitrary fixed number and $\lambda \in \mathbb{R}$. In the following lemma $(\lambda$, $u) \in \mathbb{R} \times X$ will be an arbitrary solution of (3.2).

By (H2), we can choose $b_{1} \geq 1$ such that

$$
|s| \geq b_{1} \Rightarrow|g(s)| \geq C+\beta|s| .
$$

By (1.2), we have the following

Lemma 3.1. Let (H1) hold and let $u \in X$. Then

$$
|u|_{0} \leq\left|u^{\prime}\right|_{0} \leq\left|u^{\prime \prime}\right|_{0}
$$


Lemma 3.2. Let $u$ be a solution of (3.2). Then for any $x_{0}, x_{1} \in[0,1]$,

$$
\begin{aligned}
& u^{\prime}\left(x_{1}\right)^{2}+\lambda u\left(x_{1}\right)^{2}+2 \alpha G\left(u\left(x_{1}\right)\right)=u^{\prime}\left(x_{0}\right)^{2}+\lambda u\left(x_{0}\right)^{2}+2 \alpha G\left(u\left(x_{0}\right)\right) \\
& -2 \alpha \int_{x_{0}}^{x_{1}} e(u)(s) u^{\prime}(s) \mathrm{d} s .
\end{aligned}
$$

Proof. Multiply (3.2) by $u^{\prime}$ and integrate from $x_{0}$ to $x_{1}$, then we get the desired result.

In the following, let us fix $R \in(0, \infty)$ so large that $R \geq b_{1}$ and

$$
\begin{aligned}
& g(r)+p(t, r, v)>0, \quad t \in[0,1], v \in \mathbb{R}, r>R, \\
& g(r)+p(t, r, v)<0, \quad t \in[0,1], v \in \mathbb{R}, r<-R .
\end{aligned}
$$

Lemma 3.3. There exists an increasing function $\zeta_{1}:[0, \infty) \rightarrow[0, \infty)$, such that for any solution $u$ of (3.2) with $0 \leq \lambda \leq R$ and $|u(x 0)|+\left|u^{\prime}\left(x_{0}\right)\right| \leq R$ for some $x_{0} \in[0,1]$, we have

$$
\left|u^{\prime}\right|_{0} \leq \zeta_{1}(R)
$$

Proof. Choose $x_{1} \in[0,1]$ such that $\left|u^{\prime}\right|_{0}=\left|u^{\prime}\left(x_{1}\right)\right|$. We obtain from Lemma 3.2 that

$$
\begin{aligned}
\left|u^{\prime}\right|_{0}^{2} & =u^{\prime}\left(x_{1}\right)^{2} \\
& \leq u^{\prime}\left(x_{1}\right)^{2}+\lambda u\left(x_{1}\right)^{2}+2 \alpha G\left(u\left(x_{1}\right)\right) \\
& =u^{\prime}\left(x_{0}\right)^{2}+\lambda u\left(x_{0}\right)^{2}+2 \alpha G\left(u\left(x_{0}\right)\right)-2 \alpha \int_{x_{0}}^{x_{1}} e(u)(\xi) u^{\prime}(\xi) \mathrm{d} \xi
\end{aligned}
$$

Combining this with (3.1), (3.4), it concludes that

$$
\left|u^{\prime}\right|_{0}^{2} \leq R^{2}+R^{3}+2 \Gamma(R)+2\left(C+\beta|u|_{0}\right)\left|u^{\prime}\right|_{0} \leq K(R)+2 C\left|u^{\prime}\right|_{0}+2 \beta\left|u^{\prime}\right|_{0^{\prime}}^{2}
$$

with

$$
K(R)=R^{2}+R^{3}+2 \Gamma(R) .
$$

This implies

$$
\left|u^{\prime}\right|_{0} \leq \zeta_{1}(R):=\frac{2 C+\sqrt{4 C^{2}+4(1-2 \beta) K(R)}}{2(1-2 \beta)} .
$$

Define

$$
\zeta_{2}(s)=\zeta_{1}\left(s+s^{2}\right)+1, \quad s>0 .
$$

Clearly, the function is nondecreasing.

Lemma 3.4 Let $u$ be a solution of (3.2) with $0 \leq \lambda \leq R$ and $\left|u^{\prime}\right|_{0} \geq \zeta_{2}(R)$ for some $R$ $>0$. Then, for any $x \in[0,1]$ with $|u(x)| \leq R$, we have $\left|u^{\prime}(x)\right| \geq R^{2}$.

Proof. Suppose, on the contrary that there exists $x_{0} \in(0,1)$ such that $\left|u\left(x_{0}\right)\right| \leq R$ and $\left|u^{\prime}\left(x_{0}\right)\right|<R^{2}$. Then

$$
\left|u\left(x_{0}\right)\right|+\left|u^{\prime}\left(x_{0}\right)\right|<R+R^{2} .
$$


Combining this with $\lambda \leq R<R+R^{2}$ and using Lemma 3.3, it concludes that

$$
\left|u^{\prime}\right|_{0} \leq \zeta_{1}\left(R+R^{2}\right)
$$

However, this is impossible if $\left|u^{\prime}\right|_{0} \geq \zeta_{2}(R)$.

For fixed $R>b_{1}$, let us define

$$
\chi(s):=\zeta_{2}(R)+\zeta_{2}(s), \quad s \geq 0 .
$$

Let us now consider the problem

$$
u^{\prime \prime}+\lambda u+\theta\left(\left|u^{\prime}\right|_{0} / \chi(\lambda)\right)(g(u)+e(u))=0, \quad u \in X,
$$

where $\theta: \mathbb{R} \rightarrow \mathbb{R}$ is a strictly increasing, $C^{\infty}$-function with $\theta(s)=0, s \leq 1$ and $\theta(s)=$ $1, s \geq 2$. The nonlinear term in (3.8) is a continuous function of $(\lambda, u) \in \mathbb{R} \times X$ and is zero for $\lambda \in \mathbb{R},\left|u^{\prime}\right|_{0} \leq \chi(\lambda)$, so (3.8) becomes a linear eigenvalue problem in this region, and overall the problem can be regarded as a bifurcation (from $u=0$ ) problem.

The next lemma now follows immediately.

Lemma 3.5 The set of solutions $(\lambda, u)$ of (3.8) with $\left|u^{\prime}\right|_{0} \leq \chi(\lambda)$ is

$$
\{(\lambda, 0): \lambda \in \mathbb{R}\} \cup\left\{\left(\lambda_{k}, t \phi_{k}\right): k \geq 1,|t| \leq \chi\left(\lambda_{k}\right) /\left|\phi^{\prime} k\right|_{0}\right\} .
$$

We also have the following global bifurcation result for (3.8).

Lemma 3.6 For each $k \geq 1$ and $v \in\{+,-\}$, there exists a connected set $\mathcal{C}_{k}^{v} \subset \mathbb{R} \times E$ of nontrivial solutions of (3.8) such that $\mathcal{C}_{k}^{v} \cup\left(\lambda_{k}, 0\right)$ is closed and connected and:

(i) there exists a neighborhood $N_{k}$ of $\left(\lambda_{k}, 0\right)$ in $\mathbb{R} \times E$ such that $N_{k} \cap \mathcal{C}_{k}^{v} \subset \mathbb{R} \times \Gamma_{k}^{v}$,

(ii) $\mathcal{C}_{k}^{v}$ meets infinity in $\mathbb{R} \times E$ (that is, there exists a sequence $\left(\lambda_{n}, u_{n}\right) \in \mathcal{C}_{k}^{v}, n=1,2, \ldots$, such that $\left.\left|\lambda_{n}\right|+\left|u_{n}\right|_{E} \rightarrow \infty\right)$.

Proof. Since $L^{-1}: Y \rightarrow X$ exists and is bounded, (3.8) can be rewritten in the form

$$
u=\lambda L^{-1} u+\theta\left(\left|u^{\prime}\right|_{0} / \chi(\lambda)\right) L^{-1}(g(u)+e(u)),
$$

and since $L^{-1}$ can be regarded as a compact operator from $Y$ to $E$, it is clear that finding a solution $(\lambda, u)$ of (3.8) in $\mathbb{R} \times E$ is equivalent to finding a solution of (3.9) in $\mathbb{R} \times E$. Now, by the similar method used in the proof of [1, Theorem 4.2]), we may deduce the desired result.

Since $e(u)(t) \sigma 0$ in (3.8), nodal properties need not be preserved. However, we will rely on preservation of nodal properties for "large" solutions, encapsulated in the following result.

Lemma 3.7 If $(\lambda, u)$ is a solution of (3.8) with $\lambda \geq 0$ and $\left|u^{\prime}\right|_{0}>\chi(\lambda)$, then $u \in \Gamma_{k}^{v}$, for some $k \geq 1$ and $v \in\{+,-\}$.

Proof. If $u \notin \Gamma_{k}^{v}$ for any $k \geq 1$ and $v$, then one of the following cases must occur:

Case 1. $u^{\prime}(0)=0$;

Case 2. $u^{\prime}(\tau)=u^{\prime \prime}(\tau)=0$ for some $\tau \in(0,1]$.

In the Case $1, u(t) \equiv 0$ on $[0,1]$. This contradicts the assumption $\left|u^{\prime}\right|_{0}>\chi(\lambda) \geq \zeta_{2}(\lambda)$. So this case cannot occur.

In the Case 2, we have from (3.8) that

$$
\lambda u(\tau)+\theta\left(\left|u^{\prime}\right|_{0} / \chi(\lambda)\right)(g(u(\tau))+e(u)(\tau))=0 .
$$


Since $\left|u^{\prime}\right|_{0}>\chi(\lambda)$, we have from the definition of $\theta$ that

$$
\theta\left(\left|u^{\prime}\right|_{0} / \chi(\lambda)\right)>0
$$

It follows from Lemma 3.4 that $|u(\tau)|>R \geq b_{1}$. Combining this with (3.11) and (3.3), it concludes that

$$
\lambda u(\tau)+\theta\left(\left|u^{\prime}\right|_{0} / \chi(\lambda)\right)(g(u(\tau))+e(u)(\tau)) \neq 0,
$$

which contradicts (3.10). So, Case 2 cannot occur.

Therefore, $u \in \Gamma_{k}^{v}$ for any $k \geq 1$ and $v \in\{+,-\}$.

In view of Lemmas 3.5 and 3.7, in the following lemma, we suppose that $(\lambda, u)$ is an arbitrary nontrivial solution of (3.8) with $\lambda \geq 0$ and $u \in \Gamma_{k}^{v}$, for some $k \geq 1$ and $v$.

Lemma 3.8. There exists an integer $k_{0} \geq 1$ (depending only on $\chi(0)$ ) such that for any nontrivial solution $u$ of (3.8) with $\lambda=0$ and $\chi(0) \leq\left|u^{\prime}\right|_{0} \leq 2 \chi(0)$, we have

$$
k<k_{0} \text {. }
$$

Proof. Let $x_{1}, x_{2}$ be consecutive zeros of $u$. Then there exists $x_{3} \in\left(x_{1}, x_{2}\right)$ such that $u^{\prime}\left(x_{3}\right)=0$, and hence, Lemma 3.4, (3.3), and (3.7) yield that $\left|u\left(x_{3}\right)\right|>1$. Since

$$
\begin{aligned}
2 & <\left|u\left(x_{2}\right)-u\left(x_{3}\right)\right|+\left|u\left(x_{3}\right)-u\left(x_{1}\right)\right| \\
& =\left|\left(x_{2}-x_{3}\right) u^{\prime}\left(\tau_{1}\right)\right|+\left|\left(x_{3}-x_{1}\right) u^{\prime}\left(\tau_{2}\right)\right| \\
& \leq\left(x_{2}-x_{3}\right)\left|u^{\prime}\right|_{0}+\left(x_{3}-x_{1}\right)\left|u^{\prime}\right|_{0} \\
& =\left(x_{2}-x_{1}\right)\left|u^{\prime}\right|_{0}
\end{aligned}
$$

for some $\tau_{1} \in\left(x_{3}, x_{2}\right), \tau_{2} \in\left(x_{1}, x_{3}\right)$, it follows that

$$
\left|x_{2}-x_{1}\right|>2 /\left|u^{\prime}\right|_{0}
$$

Notice that $\left|u^{\prime}\right|_{0}>\chi(0) \geq \zeta_{2}(R)$ implies that $u \in \Gamma_{k}^{v}$ for some $k \in \infty$ and $v \in\{+,-\}$, and subsequently, there exist $0<r_{1}<r_{2}<\cdots<r_{k-1}$, such that

$$
u\left(r_{j}\right)=0, \quad j=1, \ldots, k-1 .
$$

This together with (3.14) imply that

$$
1>(k-1) \cdot 2 /\left|u^{\prime}\right|_{0}
$$

and accordingly, $k<\left|u^{\prime}\right|_{0} / 2+1 \leq \chi(0)+1$.

Now let

$$
V_{R}(u)=\{x \in[0,1]:|u(x)| \geq R\}, \quad W_{R}(u)=\{x \in[0,1]:|u(x)|<R\} .
$$

Lemma 3.9. Suppose that $0 \leq \lambda \leq R$ and $\left|u^{\prime}\right|_{0} \geq \chi(R)$. Then $W_{R}(u)$ consists of at least $k$ intervals and at most $k+1$ intervals, each of length less than $2 / R$, and $V_{R}(u)$ consists of at least $k$ intervals and at most $k+1$ intervals.

Proof. Lemma 3.4 implies that $\left|u^{\prime}(x)\right| \geq R^{2}$ for all $x \in W_{R}(u)$. For any interval $I \subset W_{R}$ $(u), u$ does not change sign on $I$, say,

$$
u^{\prime}(x) \geq R^{2}, \quad x \in I .
$$

We claim that the length of $I$ is less than $2 / R$. 
In fact, for $x, y \in I$ with $x>y$, say,

$$
u(x)-u(y)=\int_{y}^{x} u^{\prime}(s) \mathrm{d} s \geq R^{2}(x-y) .
$$

Thus,

$$
x-y \leq \frac{R-(-R)}{R^{2}}=\frac{2}{R},
$$

which implies

$$
|I| \leq \frac{2}{R}
$$

The case

$$
u^{\prime}(x) \leq-R^{2}, \quad x \in I
$$

can be treated by the similar method. Since $u$ is monotonic in any subinterval containing in $W_{R}(u)$, the desired result is followed.

Lemma 3.10. There exists $\zeta_{3}$ with $\lim _{R \rightarrow \infty} \zeta_{3}(R)=0$, and $\eta_{1} \geq 0$ such that, for any $R$ $\geq \eta_{1}$, if either
(a) $0 \leq \lambda \leq R$ and $\left|u^{\prime}\right|_{0}=2 \chi(R)$, or
(b) $\lambda=R$ and $\chi(R) \leq\left|u^{\prime}\right|_{0} \leq 2 \chi(R)$,

then the length of each interval of $V_{R}(u)$ is less than $\zeta_{3}(R)$.

Proof. Define $H=H(R)$ by

$$
H(R)^{2}:=\min \{R, \min \{g(\xi) / \xi:|\xi| \geq R\}-(C / R+\beta)\},
$$

and let $\zeta_{3}(R):=2 \pi / H(R)$. By (1.4), $\lim _{R \rightarrow \infty} H(R)=\infty$, so $\lim _{R \rightarrow \infty} \zeta_{3}(R)=0$, and we may choose $\eta_{1} \geq b_{1}$ sufficiently large that $H(R)>0$ for all $R \geq \eta_{1}$.

We firstly show that

$$
|u(\tau)|>R, \quad \text { for some } \tau \in(0,1) .
$$

In fact, if $|u(x)| \leq R$ on $[0,1]$, then Lemma 3.4 yields that either

$$
u^{\prime}(x) \geq R^{2}, \quad x \in[0,1],
$$

or

$$
u^{\prime}(x) \leq-R^{2}, \quad x \in[0,1] .
$$

However, these contradict the boundary conditions (1.2), since $(\mathrm{H} 1)$ implies $u^{\prime}\left(s_{0}\right)=$ 0 for some $s_{0} \in(0,1)$. Therefore, (3.15) is valid.

Now, Let us choose $x_{0}, x_{2}$ such that either

(1) $u\left(x_{0}\right)=u\left(x_{2}\right)=R$ and $u>R$ on $\left(x_{0}, x_{2}\right)$ or

(2) $u\left(x_{0}\right)=R, x_{2}=1$ and $u>R$ on $\left(x_{0}, 1\right]$.

(the case of intervals on which $u<0$ is similar). Let

$$
I=\left[x_{0}, x_{2}\right] .
$$


By (3.8) and the construction of $H(R)$, if either (a) or (b) holds then

$$
-u^{\prime \prime}(x) \geq H(R)^{2} u(x)>0, \quad x \in I,
$$

and by Lemma 3.4, $u^{\prime}\left(x_{0}\right)>0$, and $u^{\prime}\left(x_{2}\right)<0$, if $x_{2}<1$.

Suppose, now on the contrary that $x_{2}-x_{0}>\zeta_{3}(R)$, that is, $l:=2 \pi /\left(x_{2}-x_{0}\right)<H(R)$.

Defining $x_{1}=\left(x_{0}+x_{2}\right) / 2$ and

$$
v(x)=1+\cos l\left(x-x_{1}\right), \quad x \in I,
$$

we have

$$
\begin{aligned}
& v\left(x_{0}\right)=v\left(x_{2}\right)=0, \quad v^{\prime}\left(x_{0}\right)=v^{\prime}\left(x_{2}\right)=0, \\
& v^{\prime \prime}(x)=-l^{2}(v(x)-1), \quad x \in I,
\end{aligned}
$$

and hence

$$
\begin{aligned}
0 & =\int_{x_{0}}^{x_{2}} \frac{d}{d x}\left(u^{\prime} v-u v^{\prime}\right) \mathrm{d} x \\
& =\int_{x_{0}}^{x_{2}}\left(u^{\prime \prime} v-u v^{\prime \prime}\right) \mathrm{d} x \\
& \leq \int_{x_{0}}^{x_{2}}\left(-H^{2} u v+l^{2}(v-1) u\right) \mathrm{d} x \\
& =-l^{2} \int_{x_{0}}^{x_{2}} u \mathrm{~d} x \\
& \leq-l^{2} R<0
\end{aligned}
$$

and this contradiction shows that $x_{2}-x_{0} \leq \zeta_{3}(R)$, which proves the lemma.

Now, we are in the position to prove Theorem 1.1.

Proof of Theorem 1.1 Now, choose an arbitrary integer $k \geq k_{0}$ and $v \in\{+,-\}$, and choose $\Lambda>\max \left\{\eta_{1}, \mu_{k}\right\}$ (Here, we assume $\Lambda>\eta_{1}$, so that Lemma 3.10 could be applied!) such that

$$
(k+1) \frac{2}{\Lambda}+(k+1) \zeta_{3}(\Lambda)<1 .
$$

Notice that Lemma 3.9 implies that if $\left|u^{\prime}\right|_{0} \geq \chi(\Lambda)$, then the length of each interval of $W_{\Lambda}(u)$ is less than $\frac{2}{\Lambda}$ for $0 \leq \lambda \leq \Lambda$. This together with (3.16) and Lemma 3.10 imply that there exists no solution $(\lambda, u)$ of (3.8), which satisfies either

(a) $0 \leq \lambda \leq \Lambda$ and $\left|u^{\prime}\right|_{0}=2 \chi(\Lambda)$ or

(b) $\lambda=\Lambda$ and $\chi(\Lambda) \leq\left|u^{\prime}\right|_{0} \leq 2 \chi(\Lambda)$.

Now, let us denote

$$
\begin{aligned}
B & =\left\{(\lambda, u): 0 \leq \lambda \leq \Lambda, \chi(\lambda) \leq\left|u^{\prime}\right|_{0} \leq 2 \chi(\Lambda)\right\} \\
D_{1} & =\left\{(\lambda, u): 0 \leq \lambda \leq \Lambda,\left|u^{\prime}\right|_{0}=\chi(\lambda)\right\} \\
D_{2} & =\left\{(0, u): 2 \chi(0) \leq\left|u^{\prime}\right|_{0} \leq 2 \chi(\Lambda)\right\}
\end{aligned}
$$


It follows from Lemma 3.5 that $\mathcal{C}_{k}^{v}$ "enters" $B$ through the set $D_{1}$, while from Lemma 3.7, $\mathcal{C}_{k}^{v} \cap B \subset \mathbb{R} \times \Gamma_{k}^{v}$. Thus, by Lemma 3.6 and the fact

$$
|u|_{0} \leq\left|u^{\prime}\right|_{0}
$$

$\mathcal{C}_{k}^{v}$ must "leave" $B$. (Suppose, on the contrary that $\mathcal{C}_{k}^{v}$ does not "leave" $B$, then

$$
|u|_{0} \leq\left|u^{\prime}\right|_{0} \leq 2 \chi(\Lambda)
$$

which contradicts the fact that $\mathcal{C}_{k}^{v}$ joins $\left(\mu_{k}, 0\right)$ to infinity in $\mathbb{R} \times E$.) Since $\mathcal{C}_{k}^{v}$ is connected, it must intersect $\partial B$. However, Lemmas 3.8-3.10 (together with (3.16)) show that the only portion of $\partial B$ (other than $D_{1}$ ), which $\mathcal{C}_{k}^{v}$ can intersect is $D_{2}$. Thus, there exists a point $\left(0, u_{k}^{v}\right) \in \mathcal{C}_{k}^{v} \cap D_{2}$, and clearly $u_{k}^{v}$ provides the desired solution of (1.1)-(1.2).

\section{Acknowledgements}

The authors are grateful to the anonymous referee for his/her valuable suggestions. Supported by the NSFC (No.11061030), the Fundamental Research Funds for the Gansu Universities.

\section{Author details}

${ }^{1}$ Department of Mathematics, Northwest Normal University, Lanzhou, 730070, PR China ${ }^{2}$ School of Automation and Electrical Engineering, Lanzhou Jiaotong University, Lanzhou, 730070, PR China

\section{Authors' contributions}

The authors declare that the work was realized in collaboration with same responsibility. All authors read and approved the final manuscript.

\section{Competing interests}

The authors declare that they have no competing interests.

Received: 28 April 2011 Accepted: 15 August 2011 Published: 15 August 2011

\section{References}

1. Rynne, BP: Spectral properties and nodal solutions for second-order, $m$-point, boundary value problems. Nonlinear Anal. Theory Method Appl. 67(12), 3318-3327 (2007). doi:10.1016/j.na.2006.10.014

2. Cappieto, A, Mawhin, J, Zanolin, F: A continuation approach to superlinear periodic boundary value problems. J Diff Equ. 88, 347-395 (1990). doi:10.1016/0022-0396(90)90102-U

3. Ding, T, Zanolin, F: Periodic solutions of Duffing's equation with superquadratic potential. J. Diff. Equ. 97, 328-378 (1992). doi:10.1016/0022-0396(92)90076-Y

4. Pimbley, GH Jr: A superlinear Sturm-Liouville problem. Trans. Am. Math. Soc. 103, 229-248 (1962). doi:10.1090/S00029947-1962-0138821-6

5. Cappieto, A, Henrard, M, Mawhin, J, Zanolin, F: A continuation approach to some forced superlinear Sturm-Liouville boundary value problems. Topol. Methods Nonlinear Anal. 3, 81-100 (1994)

6. Cappieto, A, Mawhin, J, Zanolin, F: On the existence of two solutions with a prescribed number of zeros for a superlinear two point boundary value problem. Topol. Methods Nonlinear Anal. 6, 175-188 (1995)

7. Ma, R, Thompson, B: Multiplicity results for second-order two-point boundary value problems with superlinear or sublinear nonlinearities. J. Math. Anal. Appl. 303(2), 726-735 (2005). doi:10.1016/j.jmaa.2004.09.002

8. Mawhin, J, Zanolin, F: A continuation approach to fourth order superlinear periodic boundary value problems. Topol. Methods Nonlinear Anal. 2, 55-74 (1993)

9. Conti, M, Terracini, S, Verzini, G: Infinitely many solutions to fourth order superlinear periodic problems. Trans. Am. Math. Soc. 356, 3283-3300 (2004). doi:10.1090/S0002-9947-03-03514-1

10. Rynne, BP: Infinitely many solutions of superlinear fourth order boundary value problems. Topol. Methods Nonlinear Anal. 19(2):303-312 (2002)

11. Rynne, BP: Global bifurcation for $2 m$ th order boundary value problems and infinitely many solutions of superlinear problems. J. Diff. Equ. 188(2), 461-472 (2003). doi:10.1016/S0022-0396(02)00146-8

12. De Coster, C, Gaudenzi, M: On the existence of infinitely many solutions for superlinear $n$th order boundary value problems. Nonlinear World. 4, 505-524 (1997)

13. Calverta, B, Gupta, CP: Multiple solutions for a super-linear three-point boundary value problem. Nonlinear Anal. Theory Method Appl. 50, 115-128 (2002). doi:10.1016/S0362-546X(01)00738-6

14. Henrard, M: Topological degree in boundary value problems: existence and multiplicity results for second-order differential equations. In: Henrard ME (ed.) Thesis. pp. 123. Université Catholique de Louvain, Belgium (1995)

15. Rabinowitz, PH: Some global results for nonlinear eigenvalue problems. J Funct Anal. 7(3), 487-513 (1971). doi:10.1016/ 0022-1236(71)90030-9 
16. Ma, R, O'Regan, D: Nodal solutions for second-order $m$-point boundary value problems with nonlinearities across several eigenvalues. Nonlinear Anal Theory Method Appl. 64, 1562-1577 (2006). doi:10.1016/j.na.2005.07.007

doi:10.1186/1687-2770-2011-14

Cite this article as: Ma et al: Infinitely many solutions to superlinear second order $m$-point boundary value problems. Boundary Value Problems 2011 2011:14.

Submit your manuscript to a SpringerOpen ${ }^{\circ}$ journal and benefit from:

- Convenient online submission

- Rigorous peer review

- Immediate publication on acceptance

- Open access: articles freely available online

- High visibility within the field

- Retaining the copyright to your article

Submit your next manuscript at $\boldsymbol{\nabla}$ springeropen.com 